\title{
Valence-Electron Resonances in Alkali-Metal Overlayers Observed via Photoemission Line-Shape Changes
}

\author{
A. Carlsson, D. Claesson, S.-Å. Lindgren, and L. Walldén \\ Physics Department, Chalmers University of Technology, 41296 Göteborg, Sweden
}

(Received 27 November 1995)

\begin{abstract}
Valence-electron resonances for $\mathrm{Na}$ overlayers on $\mathrm{Ag}(111)$ and $\mathrm{Cu}(111)$ are observed by photoemission via line-shape changes for the substrate $s, p$-band emission in a narrow range of photon energies. The effect is ascribed to interfering contributions to the photoelectron wave from the substrate and the vacuum barrier. [S0031-9007(96)00618-7]
\end{abstract}

PACS numbers: 73.20.At, 73.20.Dx, 79.60.Dp

Alkali metals adsorbed on metal surfaces attract interest as prototype examples of simple chemisorption [1] and simple metal quantum well systems [2]. In spite of the numerous studies made the electronic structure is still rather poorly characterized. So far, observations of the filled states have been limited to the discrete states that may be formed by alkali metal valence electrons in the energy range of substrate band gaps [3]. These states are observed in a photoemission spectrum as narrow peaks which are easily resolved from the low background characteristic of the substrate energy gap. More typical of metal overlayers on a metal is the formation of resonances with an energy spread due to overlap with a continuum of states in the substrate. As a result of the weak optical transition probability of a free-electron-like metal, the alkali-metal overlayer resonances are, in general, difficult to detect by photoemission. Here we show that, at least in a few cases, alkali-metal overlayer resonances can still be observed by this method, although not in the usual manner via the appearance of characteristic emission peaks. Instead, a resonance is revealed by overlayer induced line-shape changes for the emission out of substrate bulk states. For the cases discussed below, two and three atomic layers of $\mathrm{Na}$ on $\mathrm{Ag}(111)$ or $\mathrm{Cu}(111)$, the line-shape change is drastic but only in a narrow range of photon energies when the optical $k$-conservation rule selects substrate states in the energy range of the resonance. The results can be understood, we believe, as an interference in the photoemission matrix element between contributions from different spatial parts of the initial state. The bulk of the substrate gives a contribution which is characterized by a narrow emission line due to $k$-conserving optical transitions between $s, p$ bands while a broader spectrum, reflecting the width of the resonance, is generated at the interfaces and in the interior of the film.

The present observations are made by recording angle resolved photoemission spectra along the surface normal for $\mathrm{Ag}(111)$ and $\mathrm{Cu}(111)$ surfaces covered with different amounts of $\mathrm{Na}$ by evaporation of the alkali metal from a heated glass ampoule broken in situ. The sample is kept at $170 \mathrm{~K}$ by cooling its holder with liquid $\mathrm{N}_{2}$. Previous results $[4,5]$ obtained with $\mathrm{Cu}(111)$ as a substrate show that at this temperature the film grows in a layer, by atomic layer fashion, during the formation of the first four atomic layers. The present work indicates a similar growth on $\mathrm{Ag}(111)$. We therefore believe that the effect discussed below is not due to film inhomogeneities, and this is suggested also by the fact that an exact coverage of 2 monolayers (ML) is not critical for the observation. The incident light ( $p$-polarized, $40^{\circ}$ incidence angle) is obtained from a normal incidence monochromator in the MAX synchrotron radiation laboratory (BL 52). The results presented in detail below are for $2 \mathrm{ML}$ of $\mathrm{Na}$ on $\operatorname{Ag}(111)$. Similar results are obtained for $2 \mathrm{ML}$ of $\mathrm{Na}$ on $\mathrm{Cu}(111)$, but, as discussed below, the interference phenomenon of the main present interest is somewhat better resolved with $\mathrm{Ag}$ as a substrate. The effect is observed also for thicker $\mathrm{Na}$ films.

A well known feature of photoemission spectra recorded in the normal direction from the close packed surface of a noble metal is a peak due to $s, p$-band transitions across the energy gap responsible for the necks of the Fermi surface at the $L$ point of the Brillouin zone. For Ag, these transitions can be monitored by photoemission from the threshold photon energy, about $4 \mathrm{eV}$, to about $h \nu=11 \mathrm{eV}$ when the initial state energy has shifted into the $4 d$ band region $4 \mathrm{eV}$ and further below the Fermi energy. For most photon energies in this range this emission is also observed when the $\mathrm{Ag}(111)$ surface is covered by $2 \mathrm{ML}$ of $\mathrm{Na}$ (Fig. 1). However, in a narrow range of photon energies, between approximately 5.5 and $6.5 \mathrm{eV}$, the overlayer induces a drastic change of the spectrum. Here the peak is split into a doublet, which has no counterpart in the spectrum recorded for the clean substrate. For comparison, we plot in Fig. 2 the initial state energy versus photon energy for the $\mathrm{Ag}(111) /(2 \mathrm{ML} \mathrm{Na})$ sample and the clean $\mathrm{Ag}$ surface, or the Ag surface covered by a small amount of $\mathrm{Na}$, such that the work function is reduced enough that the interband transitions can also be monitored at low photon energies when the initial state is close below the edge of the $L$ gap. If $2 \mathrm{ML}$ of $\mathrm{Na}$ are deposited on $\mathrm{Cu}(111)$, a similar deviation from the dispersion observed 


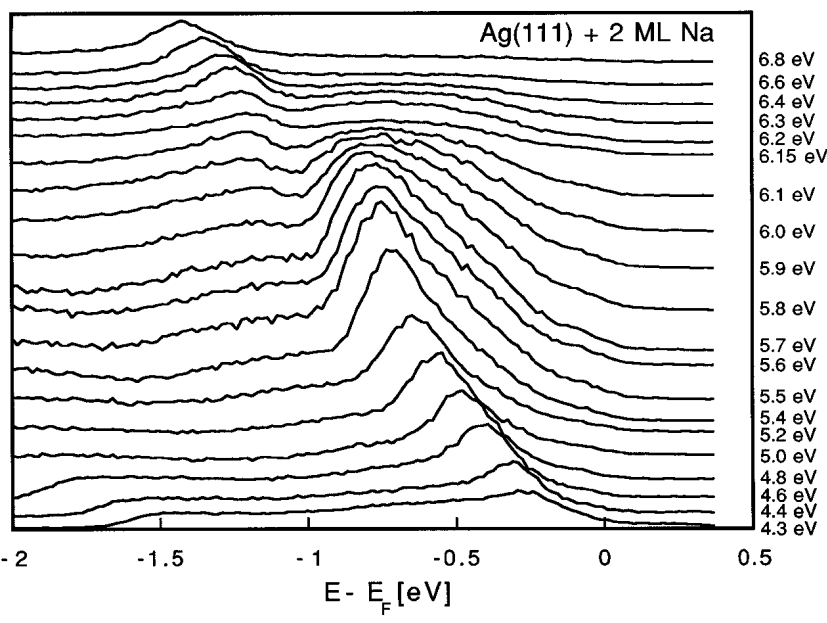

FIG. 1. Photoelectron energy spectra at different photon energies recorded along the surface normal to an $\operatorname{Ag}(111)$ crystal covered with $2 \mathrm{ML}$ of $\mathrm{Na}$. The incident light is $p$ polarized and the incidence angle is at $40^{\circ}$.

for the clean crystal is observed at nearly the same initial state energy as for Ag. The main difference is that the minimum between the two peaks is less pronounced for $\mathrm{Cu}$ and that the deviation occurs at a few tenths of an $\mathrm{eV}$ lower photon energy.

The peak splitting is accompanied by an increase of the emission intensity. The enhancement of the emission at $h \nu=6.0 \mathrm{eV}$ is evident from the two spectra shown in Fig. 3. This photon energy was also chosen to show the difference between the spectrum characteristic of the clean, or, as in Fig. 3, slightly Na covered, surface and the spectrum for $2 \mathrm{ML}$ of $\mathrm{Na}$ at a photon energy for which the doublet character is particularly clear. We note that for this photon energy the dip in the upper spectrum falls at the same energy as the peak in the lower spectrum (Fig. 3). It will be argued below that, loosely speaking, the dip in the upper spectrum is an inverted version of the peak in the lower one.

If $3 \mathrm{ML}$ of $\mathrm{Na}$ is absorbed on $\mathrm{Ag}(111)$, the observations are similar to those for $2 \mathrm{ML}$, but the deviation from the dispersion characteristic of clean $\mathrm{Ag}$ occurs at a slightly higher initial state energy. For 4 and $5 \mathrm{ML}$ there is, in addition to the dispersing $\mathrm{Ag}$ bulk peak, a peak which remains at constant initial energy $\left(E-E_{F}=-0.4\right.$ and $-0.3 \mathrm{eV}$ for 4 and $5 \mathrm{ML}$, respectively). The stationary peaks have appreciable strength only for photon energies below approximately $6 \mathrm{eV}$. At $6 \mathrm{ML}$ coverage, we observe emission from a discrete overlayer state $0.15 \mathrm{eV}$ below $E_{F}$ formed within the $\mathrm{Ag}$ band gap which extends $0.3 \mathrm{eV}$ below $E_{F}$ [6]. For $\mathrm{Cu}$, the gap extends further below $E_{F}(0.8 \mathrm{eV})$ [7], and a discrete state is already observed at a thickness of $4 \mathrm{ML}$, while for $3 \mathrm{ML}$ the observations are similar to those for 2 ML.

According to the plot shown in Fig. 2, it is as if a $0.4 \mathrm{eV}$ wide gap had been induced in the $\mathrm{Ag} s, p$ band

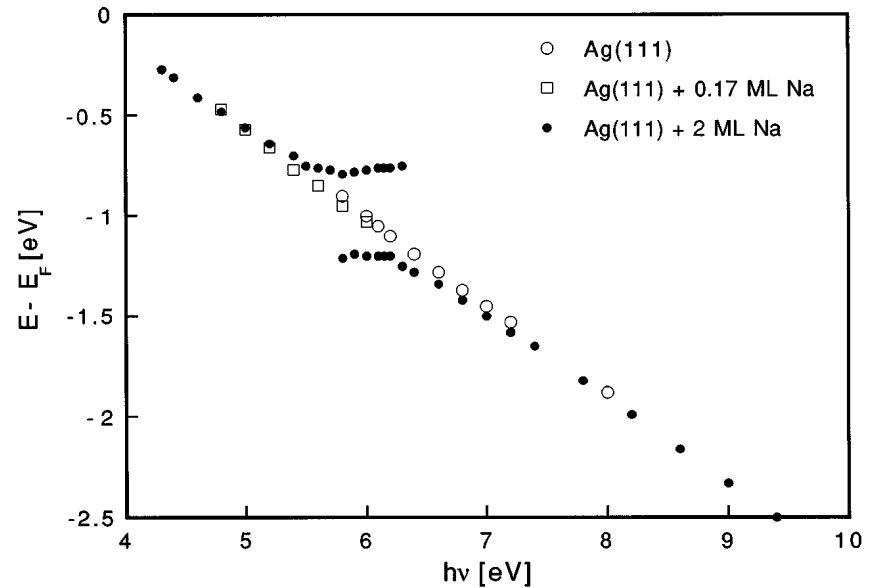

FIG. 2. Initial state energy versus photon energy for $\operatorname{Ag}(111)$ or $\operatorname{Ag}(111)$ covered with $0.15 \mathrm{ML} \mathrm{Na}$ and for $\operatorname{Ag}(111)$ covered with 2 ML of Na.

$1 \mathrm{eV}$ below $E_{F}$ as the $2 \mathrm{ML}$ thick Na overlayer is placed on top of the substrate. This would be the conclusion if the spectra were to be interpreted in the conventional manner when a photoemission peak is assumed to correspond to a state in the solid. Since no such gap is expected for the present systems, the results demonstrate the need for a different interpretation. We suggest that the apparent gap is a consequence of interfering contributions to the photoemission matrix element when the initial energies of the $\mathrm{Ag}$ bulk states involved in the $s, p$-band transitions are near an overlayer resonance.

An overlayer resonance is, in fact, expected to appear at about the initial energy of the apparent energy gap. This is demonstrated by electronic structure calculations

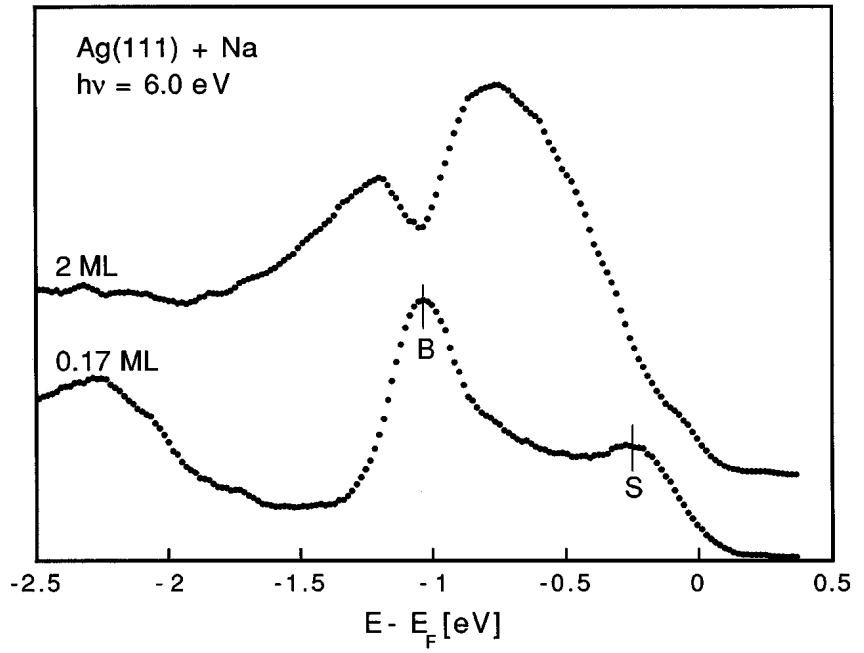

FIG. 3. Photoemission spectrum measured in the normal direction for $\mathrm{Ag}(111)$ covered with $0.15 \mathrm{ML}$ (lower spectrum) of $\mathrm{Na}$ and $2 \mathrm{ML}$ of $\mathrm{Na}$, both recorded at $6.0 \mathrm{eV}$ photon energy. The peak labeled $S$ is due to an $\operatorname{Ag}(111)$ surface state, and the peak labeled $B$ is due $s, p$-bulk band transitions. Due to the alkali deposit, $S$ appears at a lower energy and has a lower intensity than for clean $\mathrm{Ag}(111)$. 
which have been made for one or two atomic layers thick alkali films surrounded by vacuum [8,9]. As such a film is taken from vacuum and placed on a metal surface, some of the discrete states will broaden into resonances, but, as discussed previously [2], the energy is mildly affected by the transfer from vacuum to the surface.

Next, we try to explain the unusual manner in which the overlayer resonance is observed. Ordinarily, an overlayer state or resonance is found to produce an emission peak which is more or less strong depending on the photon energy [5,10]. Miller, Samsavar, and Chiang [10] observed an oscillatory emission intensity and dispersive energy upon variation of the photon energy for resonance in $\mathrm{Ag}$ overlayers thick enough that, in contrast to our case, the emission in dominated by the contribution from the overlayer. Obviously, for 2 and $3 \mathrm{ML}$ thick Na films, the emission intensity of the alkali overlayer resonances are too weak to be observed in this direct way. Their contribution to the matrix element may, however, be sufficient to be observed via the interference with a stronger photoelectron wave, which we assume is the wave emanating from the substrate $s, p$-band transitions. For the clean Ag crystal these transitions are observed as an approximately $0.25 \mathrm{eV}$ wide peak at the photon energies of present interest. The overlayer resonance and the spectrum it generates via emission at the interfaces and the interior of the film is expected to the appreciably broader than this. The dip between the two peaks of the doublet may then be the result of a destructive interference near the energy where the two emission peaks coincide.

The overall enhancement of the intensity upon deposition of $2 \mathrm{ML}$ of $\mathrm{Na}$ shown in Fig. 3 is explained by an enhanced local density of $\mathrm{Cu} s, p$ electrons close to the metal-metal interface for energies near the resonance. The enhancement is observed only for photon energies near $6 \mathrm{eV}$ when initial states in the range of the resonance are probed. This selective intensity increase is due to an enhanced amplitude for states near resonance not only in the overlayer but also in the substrate near its interface with the $\mathrm{Na}$ layer. For clean $\mathrm{Ag}(111)$ or $\mathrm{Cu}(111)$ the density of bulk band electrons will be small, close beneath the surface at energies near the low energy edge of the $L$ gap. This, as discussed in detail by Inglesfield [11], is a consequence of the difficulty of fitting a vacuum tail to the standing wave state at the zone boundary. This state, $L_{2^{\prime}}$, has maximum amplitude between the (111) planes and maximum amplitude also at the vacuum interface (if this is assumed to lie half an interlayer spacing outside the cores of the outermost atoms). Experimentally, the reduced electron density was observed by Petroff and Thiry [12] via a strong attenuation of $\mathrm{Cu}(111)$ $s, p$-band photoemission intensity as the initial state approaches the $L$ point. If an overlayer resonance appears in this energy range, the situation is reversed. By defi- nition the amplitude is then large in the overlayer, and a smooth joint at the metal-metal boundary means that the amplitude is large also on the substrate side of the interface.

If the peak splitting is to be explained by the destructive interference mentioned above, then at least one of the contributions to the matrix element must have a phase which varies rapidly across the observed doublet. This condition is satisfied by the contribution from the $s, p$ bulk band transitions in the substrate. For this part of the matrix element there is a rapid phase change, $\Delta \phi$, where $\Delta \phi=\arctan \left[\left(E-E_{0}\right) / \Delta\right]$, by $\pi$ as the energy $E$ moves across the narrow bulk peak $(2 \Delta=0.25 \mathrm{eV}$ FWHM, centered at $E_{0}$ ). Using the two band model for the substrate bands and a flat $\mathrm{Na}$ potential terminated by a step towards vacuum [2] to calculate the photoemission matrix element [13], we find that the vacuum barrier may be responsible for the wave which interferes destructively with the bulk emission. That these contributions are responsible seems reasonable since, for discrete $\mathrm{Na}$ overlayer states, it is the interference between the contributions from the vacuum barrier and the substrate potential that gives strong oscillations in the cross section when the photon energy is varied [5]. Aside from the rapid phase change for the substrate contribution, the present interference is characterized by phase shifts with a slower energy dependence, including the phase accumulation as the photoelectron wave trasverses the film and phase changes upon boundary reflections. With an adjustment of the amplitude of the surface barrier emission one also finds that, considering the simplicity of the model, the observed line-shape changes are reasonably reproduced. The simulations thus suggest that the dip of the doublet may be regarded as an antiemission line associated with an inversion of the bulk band emission peak due to the interference with a weaker and broader emission from the surface.

While the present indirect way of observing a resonance does not allow a precise determination of its energy, some information is available from the line shape. We consider the upper spectrum in Fig. 3. This dip between the two peaks marks the energy for out-of-phase contributions to the photoemission matrix element. If the peak observed for the substrate contribution (lower spectrum in Fig. 3) had coincided with the center of the overlayer resonance, a symmetric doublet would be expected. The observed asymmetry (Fig. 3) means that the center of the resonance falls on the high energy side of the dip in the upper spectrum.

Finally, we note that the present work does not provide a general solution to the problem of observing simple metal quantum well resonances. In the case of $2 \mathrm{ML}$ of $\mathrm{Na}$ on $\mathrm{Ag}(111)$ or $\mathrm{Cu}(111)$, for example, it is easy to see that there should exist one more filled resonance centered, for $k_{\|}=0$, around $2 \mathrm{eV}$ below $E_{F}$. Since the spectra give no evidence for the resonance, this appears to be one 
instance when the otherwise rather generally applicable photoemission method is not successful.

In summary, we find that alkali overlayer resonances, which give a low photoemission intensity and are therefore not easily observed as separate emission peaks, may be detected via line-shape changes of substrate emission. For $2 \mathrm{ML}$ of $\mathrm{Na}$ on $\mathrm{Ag}(111)$ or $\mathrm{Cu}(111)$ the observed, drastic line-shape change is ascribed to the interference between substrate bulk emission and emission due to the vacuum barrier.

Financial support from the Wallenberg Foundation and from the Swedish Natural Science Research Council is gratefully acknowledged.

[1] The Physics and Chemistry of Alkali Metal Adsorption, edited by A. M. Bradshaw, H.P. Bontzel, and G. Ertl (Elsevier, New York, 1989).
[2] S.-Å. Lindgren and L. Walldén, Phys. Rev. B 38, 3060 (1988).

[3] S.-Å. Lindgren and L. Walldén, Solid State Commun. 34, 671 (1980).

[4] A. Carlsson, S.-Å. Lindgren, C. Svensson, and L. Walldén, Phys. Rev. B 50, 8926 (1994).

[5] A. Carlsson, D. Claesson, S.-Å. Lindgren, and L. Walldén, Phys. Rev. B 52, 11144 (1995).

[6] L. Walldén and T. Gustafsson, Phys. Scr. 6, 73 (1972).

[7] I. Lindau and L. Walldén, Phys. Scripta 3, 77 (1971).

[8] E. Wimmer, J. Phys. F 13, 2313 (1983).

[9] J. C. Boettger and S. B. Trickey, Phys. Rev. B 45, 1363 (1992).

[10] T. Miller, A. Samsavar, and T.-C. Chiang, Phys. Rev. B 50, 17686 (1994).

[11] J. E. Inglesfield, Rep. Prog. Phys. 45, 223 (1982).

[12] Y. Petroff and P. Thiry, Appl. Opt. 19, 3957 (1980).

[13] For the procedure, see Kenneth W.-K. Shung and G.D. Mahan, Phys. Rev. B 38, 3856 (1988). 